\title{
Time-Slotted Optical OV-CDMA Network Using a Fair QoS-Based Resource Management Algorithm
}

\author{
Robert Raad \\ Faculty of Sc. and Eng. \\ Laval University, Canada \\ robert.raad.1@ulaval.ca
}

\author{
Elie Inaty \\ Faculty of Eng. \\ University of Balamand, Lebanon \\ elie.inaty@balamand.edu.lb
}

\author{
Paul Fortier \\ Faculty of Sc. and Eng. \\ Laval University, Canada \\ fortier@gel.ulaval.ca
}

\author{
Hossam M. H. Shalaby \\ Faculty of Eng. \\ University of Alexanderia, Egypt \\ shalaby@ieee.org
}

\begin{abstract}
In this work we develop a fair resource allocation scheme for a multi-class time-slotted optical overlapped codedivision multiple-access (OV-CDMA) network. The resource management scheme is fair in the sense that the users have their power and rate allotted according to their quality of service $(\mathrm{QoS})$ requirements. In addition, no class of users can dominate over the other classes. A unified framework is proposed, which consists of finding a single control parameter based on which the optimal transmission rate and power are obtained for every class of users. Analytical results show that the maximum transmission rate for a given class of users is only achievable when the users in that class are transmitting with the highest possible laser transmission power. In addition, we have demonstrated that the optimal transmission rates can be obtained via the solution of the rate characteristic polynomial.
\end{abstract}

Keywords- Optical OV-CDMA, multirate, multiclass, resource allocation, overlapping coefficient, time-slotted network.

\section{INTRODUCTION}

Many optical networks have been deployed with the aim of accommodating novel information-services generated by modern communication terminals like high-speed personal computers, FTTX networks, and others [1]. Those services require a huge amount of bandwidth, and multirate transmission capabilities. In this regard, we believe that this work can contribute to this research trend.

Resource allocation schemes are necessary to enhance the performance of the optical network in order to guarantee successful data transmission and satisfy users' requirements. For instance, a nonlinear programming power control algorithm has been proposed in [3]. In [6], a power control algorithm, based on optical power selector has been inspected for a multirate optical DS-CDMA system. In addition, an adaptive overlapped pulse-position modulator, employed to create multirate and multi-quality transmission schemes, has been investigated in [7]. Moreover, the power control problem is also addressed in [8] for temporal prime coded OCDMA system. Lately, we have proposed a novel hybrid power/rate control algorithm for the optical overlapped CDMA (OVCDMA) system [3]. The problem of this strategy is that it is not fair in the sense that there is at most one class of users that has an overlapping coefficient between zero and full overlap, and the remaining classes either transmit with full overlap or with no overlap. In addition, the model used in [3] did not support a time-slotted packetized network configuration as the one proposed in [4].

The first contribution of this work is to suggest a unified framework for allocating and controlling the transmission rate and power for an OV-CDMA-based optical network using matrix analysis. This approach is general in the sense that it can be applied for any expression of the system capacity. In addition, it is generalized to a time-slotted packetized system. The second contribution is the reduction of the optimization search space into one dimensional space. This target guaranties the simplicity of the algorithm, which is an important requirement in optical networks. Our third contribution is to solve the problem of unfairness in the resource allocation strategy encountered in [3].

The rest of the work is organized as follows. In Section II, we present the system model. The performance of the network at the physical layer has been discussed in Section III. In Section IV, we investigate the proposed resource allocating scheme. In Section V, we define and we analyze the network capacity. Simulation results and discussion are provided in Section VI. Finally, we close our work with a conclusion in Section VII.

\section{SYSTEM MODEL}

Consider a multiclass time-slotted OV-CDMA system in an optical packet network in which $K$ users are connected in a star topology as the one proposed in [4]. The users are assigned different optical code signatures of equal processing gain (PG) $G$ and are distributed over $S$ classes. Each class- $j, \forall$ $j \in\{0,1, \ldots, S-1\}$, is characterized by its own and unique QoS, so that class- $j$ have its proper transmission power $P_{j}$ and overlapping coefficient $\varepsilon_{j}$ or equivalently its own transmission rate $R_{j}$. The users transmit their coded bits at a given time slot in the form of packets of nominal length of $L$ bits. However, due to the overlapping process, the maximum number of bits which a user can transmit per packet exceeds $L$ as presented in [4] and shown in Fig. 1. This figure illustrates an example of the overlapping process in a packet time slot. In this example, the packet length is $L=2$, and the PG is $G=5$. When the 
overlapping coefficient is $\varepsilon_{s}=3$ as shown in Fig. 1a), the transmission rate is increased to three bits per packet. On the other hand, Fig. 1b) shows the case where $\varepsilon_{s}=4$. Accordingly, the transmission rate is six bits per packet. Note that $\lambda_{i}$ represents the assigned wavelength in the $i^{\text {th }}$ time instance.

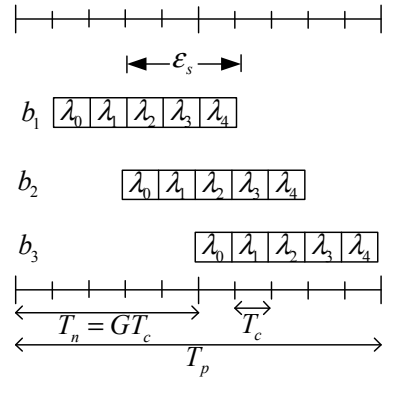

a)

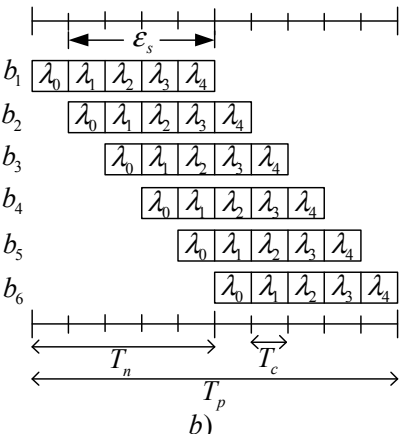

b)
Fig. 1: Optical OV-CDMA packet model of a single user in a given time slot. a) $\varepsilon_{s}=3$, b) $\varepsilon_{s}=4$

Let $0 \leq \varepsilon_{j} \leq G-1, \forall j \in\{0,1, \ldots, S-1\}$, be the overlapping coefficient and $X_{b, j}$ to be the total number of overlapped bits in a packet time slot The expression of $X_{b, j}$ can be found in [4] and it is given by

$$
X_{b, j}=\left\lfloor\frac{L G-\varepsilon_{j}}{G-\varepsilon_{j}}\right\rfloor
$$

Consequently, the achievable transmission rate of class- $j$ users is given by

$$
R_{j}=\frac{X_{b, j}}{L} R_{n}
$$

where $R_{n}$ is the nominal rate.

\section{SYSTEM PERFORMANCE}

We are interested in evaluating the average SIR per bit in a given time slot for every class- $j$, which has its own power and overlapping coefficient and supporting $K_{j}$ users. Then, the average SIR, as derived in [4], is given by

$$
\operatorname{SIR}_{j}(\varepsilon, \mathrm{P})=\frac{P_{j} G^{2}}{\left(K_{j}-1\right) P_{j} \bar{\sigma}_{I_{k_{j}}}^{2}\left(\varepsilon_{j}\right)+\sum_{\substack{s=0 \\ s \neq j}}^{S-1} \sum_{k=1}^{K_{s}} P_{s} \bar{\sigma}_{I_{k_{s}}}^{2}\left(\varepsilon_{s}\right)+\sigma_{n}^{2}}
$$

where $\varepsilon$ and $\mathrm{P}$ are vectors of the overlapping coefficients and the transmission power of all classes, respectively. $\bar{\sigma}_{I_{k j}}^{2}$ is the average multiple access interference (MAI) power imposed on the desired bit by a class- $j$ user in a given time slot [4], and it is computed as

$$
\begin{aligned}
& \bar{\sigma}_{I_{k_{j}}}^{2}= \\
& 2 \sum_{i=0}^{X_{b, j}-X_{r, j}-1}\left[\sum_{v=-i}^{-1} H_{v}^{2}\left(0, q_{v}^{(i)}\right)+\sum_{v=0}^{X_{r, j}} H_{v}^{2}\left(q_{v}^{(i)}, G\right)\right] \frac{\left(X_{b, j}-X_{r, j}\right)}{X_{b, j}} \\
& +\sum_{i=X_{b, j}-X_{r, j}}^{X_{r, j}-1}\left[\sum_{v=-i}^{-1} H_{v}^{2}\left(0, q_{v}^{(i)}\right)+\sum_{v=0}^{X_{b, j}-i-1} H_{v}^{2}\left(q_{v}^{(i)}, G\right)\right] \frac{\left(2 X_{r, j}-X_{b, j}\right)}{X_{b, j}} \\
& , \quad X_{b, j}-2 X_{r, j} \leq 0
\end{aligned}
$$

and

$$
\begin{aligned}
& \bar{\sigma}_{I_{k_{j}}}^{2}= \\
& 2 \sum_{i=0}^{X_{r, j}-1}\left[\sum_{v=-i}^{-1} H_{v}^{2}\left(0, q_{v}^{(i)}\right)+\sum_{v=0}^{X_{r, j}} H_{v}^{2}\left(q_{v}^{(i)}, G\right)\right] \frac{X_{r, j}}{X_{b, j}} \\
& +\sum_{i=X_{r, j}}^{X_{b, j}-X_{r, j}-1}\left[\sum_{v=-X_{r, j}}^{-1} H_{v}^{2}\left(0, q_{v}^{(i)}\right)+\sum_{v=0}^{X_{r, j}} H_{v}^{2}\left(q_{v}^{(i)}, G\right)\right] \frac{\left(X_{b, j}-2 X_{r, j}\right)}{X_{b, j}} \\
& , \quad X_{b, j}-2 X_{r, j}>0
\end{aligned}
$$

where $X_{r, j}=\left\lceil\varepsilon_{j} /\left(G-\varepsilon_{j}\right)\right\rceil$ as computed in [4]. In addition, $H_{v}^{2}\left(0, q_{v}^{(i)}\right)$ and $H_{v}^{2}\left(q_{v}^{(i)}, G\right)$ are the partial period Hamming correlation functions [4]. $\sigma_{n}^{2}$ represents the power of the other sources of noise modeled as Gaussian noise. It involves thermal noise, shot noise, dark current, and surface leakage current.

Assuming one-coincidence sequences with $F$ available wavelengths, we can get the average value of $\bar{\sigma}_{I_{k_{j}}}^{2}$ in terms of the number of bits per packet as follows:

$\bar{\sigma}_{I_{k_{j}}}^{2}= \begin{cases}\frac{-\frac{1}{3}\left(A_{4} X_{b, j}^{4}+A_{3} X_{b, j}^{3}+A_{2} X_{b, j}^{2}+A_{1} X_{b, j}+A_{0}\right)}{F(L-1)^{3}\left(X_{b, j}-1\right) X_{b, j}}, & L \leq \frac{3 X_{b, j}}{X_{b, j}+2} \\ \frac{\left(B_{3} X_{b, j}^{3}+B_{2} X_{b, j}^{2}+B_{1} X_{b, j}+B_{0}\right)}{F(L-1)^{3} X_{b, j}}, & L>\frac{3 X_{b, j}}{X_{b, j}+2}\end{cases}$

where $A_{i}$ for $0 \leq i \leq 4$ and $B_{j}$ for $0 \leq j \leq 3$ are constant coefficients that are function of the OV-CDMA system parameters $G, F$, and $L$. Their values are shown in the appendix.

We consider that the users are dispersed among classes according to a given distribution with a probability mass function (PMF) $p^{(j)}$ such that $\sum_{j=0}^{S-1} p^{(j)}=1$. Therefore, the total number of users in class- $j$ is given by $K_{j}=K p^{(j)}$ for $0 \leq j \leq S-1$.

\section{Resource Allocation Strategy}

Our strategy is based of the assumption that the QoS of class $-j$ in the presence of other classes should meet the requirement 


$$
S I R_{j} \geq \beta_{j}
$$

We define the solitary class $-j$ SIR as the desired signal power over the power of a class- $j$ interferer. It is given by

$$
\gamma_{j}=\frac{G^{2}}{\bar{\sigma}_{I_{k j}}^{2}}
$$

In addition, we define the nominal signal-to-noise ration (SNR) that is common to all classes as

$$
\gamma_{n}=\frac{G^{2}}{\sigma_{n}^{2}}
$$

Using the definitions of the class solitary SIR in (8) and the nominal SNR in (9), the minimum requirements in (7) gives $S$ linear equations in the laser transmission power of each class as follows,

$$
\sum_{s=0}^{S-1} \frac{K_{s}}{\gamma_{s}} P_{s}-\left(\frac{1}{\gamma_{j}}+\frac{1}{\beta_{j}}\right) P_{j}+\frac{1}{\gamma_{n}}=0, \forall 0 \leq j \leq S-1
$$

The solution of (10) represents the power allocation for all classes. Let $\Gamma=\operatorname{diag}\left(\frac{1}{\gamma_{0}}, \cdots, \frac{1}{\gamma_{S-1}}\right), \mathcal{B}=\operatorname{diag}\left(\frac{1}{\beta_{0}}, \cdots, \frac{1}{\beta_{S-1}}\right)$, and $\mathcal{K}=\operatorname{diag}\left(K_{0}, \ldots, K_{S-1}\right)$ be $S \times S$ diagonal matrices. $\mathbf{P}=\left(P_{0}, \ldots, P_{S-1}\right)^{T}$ is the vector of the unknown transmission powers of the $S$ classes, and $\mathbf{e}_{S \times 1}$ is a unity vector. Also, let $\mathbf{N}_{D}=\Gamma+\mathcal{B}, \quad \mathbf{M}_{D}=\left(\mathbf{N}_{D}-\mathcal{B}\right) \mathcal{K}=\Gamma \mathcal{K}$. Then, the linear system in (10) can be reduced to $(-\mathcal{M}) \mathbf{P}=\mathbf{e} / \gamma_{n}$, where $\mathcal{M}^{\mathrm{T}}=\mathbf{M}_{D} \mathbf{e e}^{T}-\mathbf{N}_{D} \quad$ is the system-performance matrix. Thereby, the power vector is given by

$$
\mathbf{P}=\frac{1}{\gamma_{n}}\left(-\mathcal{M}^{-1}\right) \mathbf{e}
$$

Applying the Sherman-Morrison formula to find the inverse [9], we obtain

$$
\left(\mathbf{N}_{D}-\mathbf{M}_{D} \mathbf{e e}^{T}\right)^{-1}=\left(\mathbf{I}+\frac{1}{\zeta} \mathbf{N}_{D}^{-1} \mathbf{M}_{D} \mathbf{e e}^{T}\right) \mathbf{N}_{D}^{-1}
$$

where I is an $S \times S$ identity matrix. This implies that

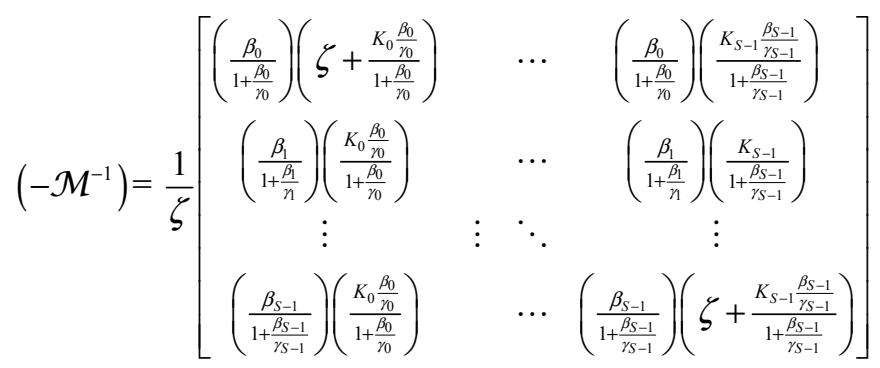

where

$$
\zeta \equiv \sum_{s=0}^{S-1}\left[\frac{K_{s}}{1+\beta_{s} / \gamma_{s}}\right]-(K-1), \zeta>0
$$

Substituting (12) in (11) we get

$$
\mathbf{P}=\frac{1}{\zeta \gamma_{n}}\left(\frac{\beta_{0}}{1+\beta_{0} / \gamma_{0}}, \cdots, \frac{\beta_{S-1}}{1+\beta_{S-1} / \gamma_{S-1}}\right)^{T}
$$

When $\zeta=0$, the optical intensity is extremely high, which leads to nonlinear effects and cause severe degradation of the signal. To avoid this situation, we impose constraints on the power of each class of users. Therefore, we consider that the optical power is upper bounded by $\Pi=\left(\pi_{0}, \ldots, \pi_{S-1}\right)^{T}$ such that

$$
P_{j} \leq \pi_{j}, \quad \forall \quad 0 \leq j \leq S-1
$$

Lemma 1: The linear system in (10) constrained by (16) has a solution if and only if the solitary class- $j$ SIR, $\gamma_{j}$, is lower bounded by

$$
\gamma_{j}^{*}=\frac{(K-1) \pi_{j}}{\sum_{s=0}^{S-1} \frac{K_{s} \pi_{s}}{\beta_{s}}-(K-1) \frac{\pi_{j}}{\beta_{j}}-\frac{1}{\gamma_{n}}}
$$

Proof: Omitted due to the limited permitted space

Lemma 2: For a given value of $\pi_{j}$ and $\beta_{j}, \gamma_{j}^{*}$ in (17) always exists $\forall 0 \leq j \leq S-1$.

Proof: Omitted due to the limited permitted space

In this work, the power allocation is assumed to be fair among all classes, which means that no class can dominate the others due to a high power allocation. Hence, let the ratio $\pi_{j} / \beta_{j}$ be arbitrarily identical for all classes. Thus, let $\alpha$ be a control parameter whose role is to manage the power level that is necessary to satisfy the required QoS and to achieve a rate augmentation whenever possible while maintaining the fairness criteria. It is given by

$$
\frac{\pi_{j}}{\beta_{j}}=\frac{\alpha}{\gamma_{n}}, \forall 0 \leq j \leq S-1
$$


The domain of $\alpha$ can be obtained from Lemma 2. The optimal value of $\alpha$ should be the one that maximizes the network capacity. On the other hand, substituting (18) in (17) yields

$$
\gamma_{j}^{*}=\frac{\alpha}{\alpha-1}(K-1) \beta_{j}
$$

\section{A. Rate Allocation and Control Scheme}

Note that, as $\gamma_{j}$ increases, $\bar{\sigma}_{I_{k_{j}}}^{2}$ decreases. This, in turn, results in a reduction of the number of overlapping bits per packet, and hence a reduction of the transmission rate. From (17), we define the rate characteristic polynomial as

$$
\rho\left(R_{j}\right) \equiv \bar{\sigma}_{I_{k_{j}}}^{2}-\frac{G^{2}}{\gamma_{j}^{*}}
$$

It is clear that because $\gamma_{j} \geq \gamma_{j}^{*}, \rho\left(R_{j}\right)$ is always negative. Therefore, the admissible region of the transmission rate is given by

$$
\mathscr{R}=\left\{\begin{array}{l}
R_{j}: R_{n} \leq R_{j} \leq \frac{(L-1) G+1}{L} R_{n}, \text { s.t } \rho\left(R_{j}\right) \leq 0, \\
\forall j \in\{0,1, \ldots, S-1\}
\end{array}\right\}
$$

Lemma 3: Let $\beta^{(\ell)}$ and $\beta^{(u)}$ be the lower and the upperbound QoS, respectively. They are given by

$$
\beta^{(\ell)}=\frac{G^{2}(1-1 / \alpha)}{(K-1) \max \left\{\bar{\sigma}_{I_{k_{j}}}^{2}\right\}}, \beta^{(u)}=\frac{G^{2}(1-1 / \alpha)}{(K-1) \min \left\{\bar{\sigma}_{I_{k_{j}}}^{2}\right\}}
$$

The maximum admissible transmission rate of class- $\mathrm{j}$ users is given by the highest admissible root of the ratecharacteristic polynomial when $\beta^{(\ell)} \leq \beta_{j} \leq \beta^{(u)}$, and it is the maximum possible rate $R^{(u)}$ of the optical OV-CDMA system when $\beta_{j}<\beta^{(\ell)}$. The system has no feasible solution when $\beta_{j}>\beta^{(u)}$.

Proof: Omitted due to the limited permitted space

\section{B. Power Allocation and Control Scheme}

The optimal transmission power for every class of users is obtained throughout the following lemma.

Lemma 4: The optimal transmission power of users in any class- $\mathrm{j}$, transmitting at the maximum admissible rate $R_{j}^{*}$, is given by

$$
P_{j}^{*}= \begin{cases}\pi_{j}=\alpha \frac{\beta_{j}}{\gamma_{n}}, & \text { for } \beta^{(\ell)} \leq \beta_{j} \leq \beta^{(u)} \\ \frac{\beta_{j}}{1+\beta_{j} / \gamma^{(\ell)}} & <\pi_{j}, \text { for } \beta_{j}<\beta^{(\ell)}\end{cases}
$$

and $P_{j}^{*}$ is not feasible for $\beta_{j}>\beta^{(u)}$.

Proof: Omitted due to the limited permitted space

Observation: When $\beta_{j}<\beta^{(\ell)}$, the resource allocation is uncontrollable. It results directly from Lemma 3 and Lemma 4 that both $R_{j}^{*}$ and $P_{j}^{*}$ are independent of the control parameter $\alpha$. Therefore, both resources are uncontrollable.

In light of the above observation, we adopt $\beta^{(\ell)} \leq \beta_{j} \leq \beta^{(u)}$ as the QoS operating interval for the remaining analysis. Thus, Lemmas 3 and Lemma 4 indicate that any class- $j$ user in the OV-CDMA packet-network can realize its highest transmission rate when the optical transmission power is at the maximum level (i.e. $\pi_{j}$ ).

\section{CAPACITY ANALISIS}

One of the challenges that exists usually in resource allocation problems is the model of the expression of the network throughput or the actual network capacity that reflects real situations [2]. In our case, it is logical to model the capacity as the ratio of the total transmission rate to the total transmission power which is given by

$$
C=\frac{\sum_{j} p^{(j)} R_{j}^{*}(\alpha)}{\sum_{j} p^{(j)} P_{j}^{*}(\alpha)}=\frac{\sum_{j} K_{j} R_{j}^{*}(\alpha)}{\sum_{j} K_{j} P_{j}^{*}(\alpha)}
$$

where $R_{j}^{*}(\alpha)$ is the transmission rate for class- $j$ users given by the solution of the rate characteristic polynomial in (20) and obtained by Lemma 3. On the other hand, $P_{j}^{*}(\alpha)$ is the laser transmission power for class- $j$ users given in (22) and obtained by Lemma 4 . If we consider $R_{j}^{*}$ normalized by $R_{n}$, then $C$ can be seen as the per user network capacity normalized by $R_{n}$ per unit of power. Our proposed criterion consists of finding the optimal control parameter $\alpha^{*}$ that maximizes $C(\alpha)$ such as

$$
\alpha^{*}=\arg \max _{\alpha>1}\{C(\alpha)\}
$$

As a result, $P_{j}^{*}\left(\alpha^{*}\right)$ and $R_{j}^{*}\left(\alpha^{*}\right)$ are optimally controlled via $\alpha^{*}$. 
Furthermore, we define the nominal capacity when all users transmit at nominal rate $R_{n}$ as

$$
C_{n}=\frac{R_{n}}{\sum_{j} p^{(j)} P_{j}^{*}}=\frac{R_{n}}{\alpha \sum_{j} p^{(j)} \beta_{j}} \gamma_{n}
$$

The control parameter is obtained as $\alpha_{n}^{*}=\arg \max _{\alpha>1}\left\{C_{n}(\alpha)\right\}$. This implies $\alpha_{n}^{*}=\min (\alpha>1)$.

\section{NUMERICAL RESULTS AND DISCUSSION}

In this section, the analytical results are compared to the numerical ones obtained using a numerical search method. This method is based on the quasi-Newton method [10]. The numerical results show a total agreement with the analytical ones and validate our analysis.

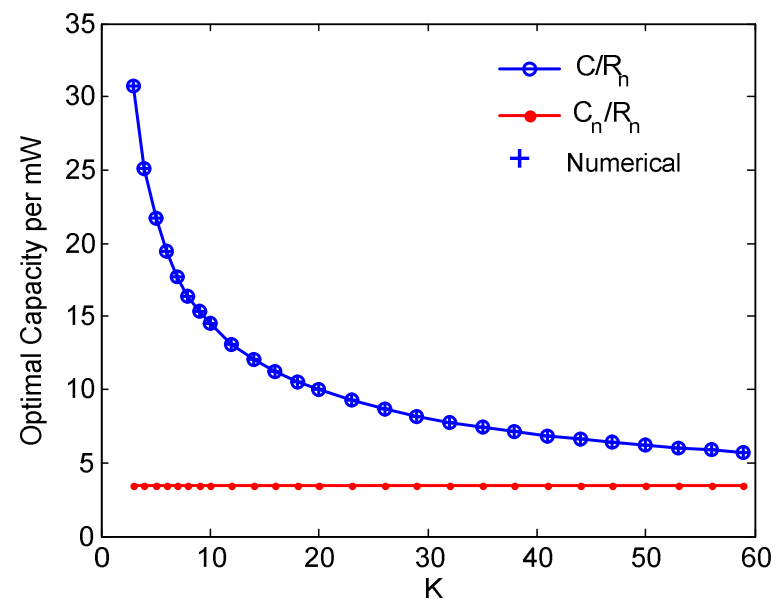

Fig. 2: The improved and the nominal capacities, normalized to $R_{n}$.

We consider a three-class system. The users are distributed among the classes as follows: $40 \%$ in class- $0,30 \%$ in class- 1 , and $30 \%$ in class- 2 . All the users are assigned codes of fixed PG $G=61$ through which they can generate packets of length $L=1024$ bits per time slot. Finally, we select $\gamma_{n}=15 \mathrm{~dB}$ common to all classes. Assume that QoS requirements are $\beta_{0}$ $=4 \mathrm{~dB}, \beta_{1}=8 \mathrm{~dB}$, and $\beta_{2}=12 \mathrm{~dB}$ for class -0 , class -1 and class-2, respectively.

Fig. 2 illustrates the optimal capacity $C$ versus the nominal capacity $C_{n}$ per active user when the number of users increases. The figure shows clearly the improvement of the optimized capacity per user with respect to the nominal one which can be seen as a lower bound. As $K$ increases, this capacity gets reduced until it eventually reaches the nominal one.

The optimal transmission power of each class of users as a function of $K$ is shown in Fig. 3. We observe that the allocated transmission power per user in each class is fairly chosen for each class of users according to its QoS requirement. Class-2 users transmit with the highest power because they require the highest QoS. In addition, users always transmit with the highest power $\pi_{j}$ which validate Lemma 4 .

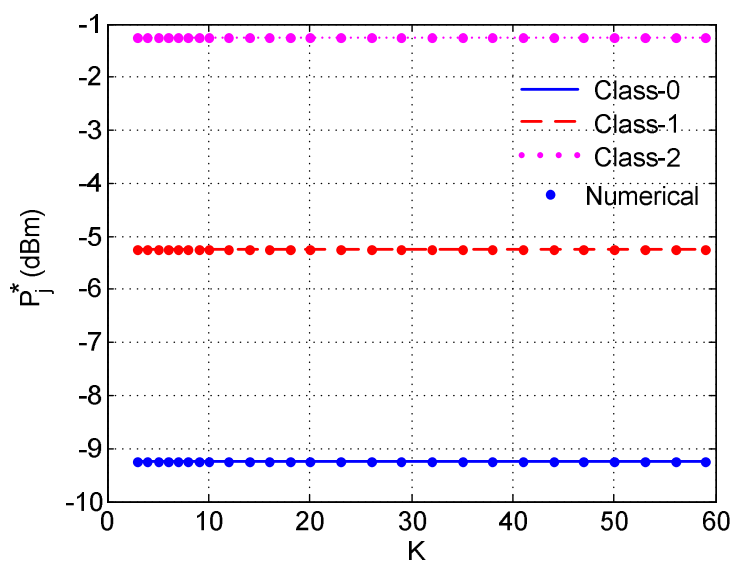

Fig. 3: The optimal transmission power versus the total number of users.

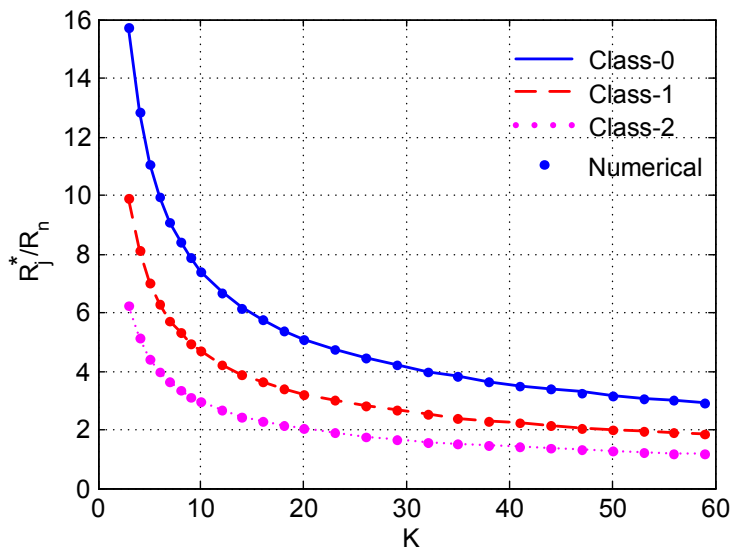

Fig. 4: The normalized optimal transmission rate of class- $j$ users versus the total number of users.

The optimal transmission rates for each class of users are depicted in Fig. 4. Using our proposed algorithm, it is clear that there is an increase in the transmission rate of each class of users. All classes can simultaneously transmit at rates varying between the lower and the upper bound cutoff rates of the OVCDMA system. As $K$ increases, each user is required to diminish the transmission bit rate to reduce the MAI effect in the optical channel.

The power control versus the rate allocation of class-1 is revealed in Fig. 5. Low rate users are illuminated with high power since they transmit with a high QoS. As the allotted transmission rate increases, the corresponding supplied power decays rapidly because the QoS has been relaxed. Moreover, when $K$ increases, less power is allowed per user and the maximum allowable increase in the transmission rate above the nominal rate is smaller. 


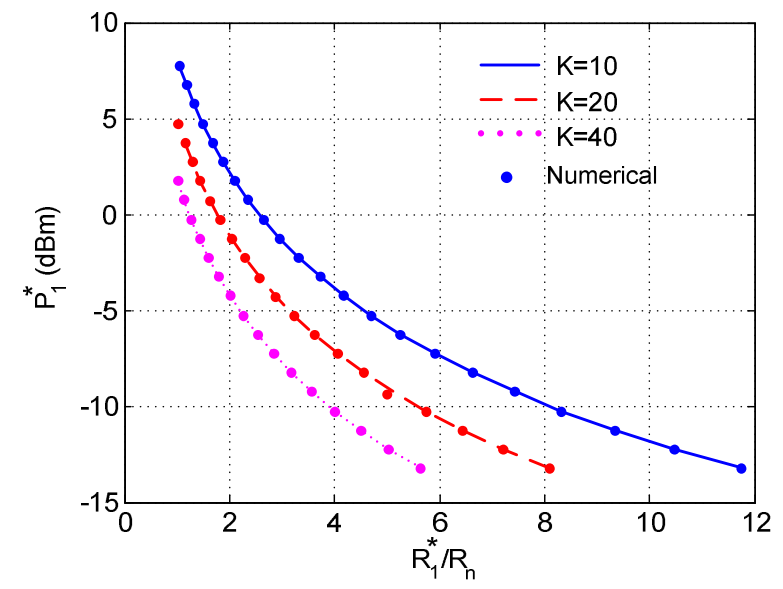

Fig. 5: The optimal transmission power allocation as a function of the normalized optimal transmission rate of class-1 users.

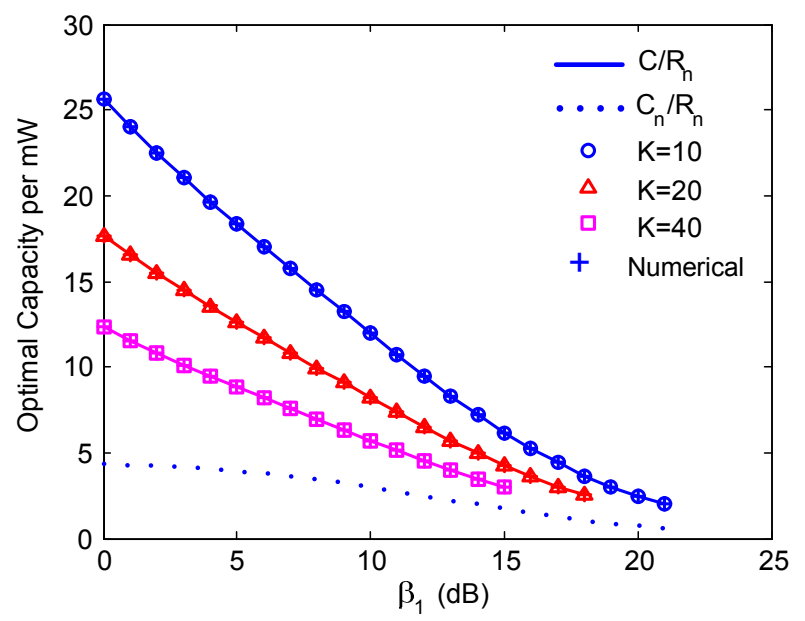

Fig. 6: The normalized optimal user's capacity versus the QoS variation of class-1.

The normalized optimal network capacity per user is depicted in Fig. 6. The improvement of the capacity with respect to the nominal capacity is obvious for different network conditions. Such an improvement diminish when the number of active users increases, and/or when the demand on QoS increases.

\section{CONCLUSION}

We proposed a fair resource allocation scheme for a multiclass time-slotted optical OV-CDMA-based packet networks.. The Sherman-Morrison's matrix inversion lemma was used to solve the power allocation problem in closed form. A ratecharacteristic polynomial has been derived and solved for the optimal transmission rate allocation. The resources are optimally allocated as functions of a single control parameter, which helps to reduce the search space into one dimensional space. A numerical search method was employed to validate the analytical results. Both numerical and analytical results proved that our resource allocation scheme is simple to implement and offers a substantial improvement to the system capacity.

\section{APPENDIX}

Polynomial coefficients in Section III:

$$
\begin{aligned}
A_{4}= & G L^{4}-12 G L^{3}+51 G L^{2}-90 G L+54 G, \\
A_{3}= & 5 G L^{4}-(42 G+6 F) L^{3}+(102 G+30 F) L^{2} \\
& -(54 G+48 F) L-27 G+24 F, \\
A_{2}= & 8 G L^{4}-(34 G+6 F) L^{3}-(G-6 F) L^{2} \\
& +(60 G+24 F) L-9 G-24 F, \\
A_{1}= & 4 G L^{4}+(8 G+6 F) L^{3}-(40 G+30 F) L^{2}+(12 G+24 F) L, \\
A_{0}= & (8 G+6 F) L^{3}-(4 G+6 F) L^{2}, B_{3}=G L^{2}-6 G L+9 G, \\
B_{2}= & 3 G L^{2}-(6 G-2 F) L-9 G-2 F, \\
B_{1}= & -4 F L^{2}+(12 G+4 F) L, B_{0}=2 F L^{3}-(4 G+2 F) L^{2}
\end{aligned}
$$

\section{REFERENCES}

[1] L. G. Kazovsky, W. Shaw, D. Gutierrez, N. Cheng, and S. Wong, "Nextgeneration Optical access networks," IEEE J. of Lightwave Technolgy., vol. 25, no. 11, pp. 3426 - 3442, Nov. 2007.

[2] D. Zhang, S. Oh, and N. T. Sindhushayana, "Optimal resource allocation for data service in CDMA reversed link," IEEE Transactions on Wireless Comm., vol. 6, no. 10, pp. 3648-3656, Oct. 2007.

[3] R. Raad, E. Inaty, P. Fortier, and H. M. H. Shalaby, "Optimal resource allocation scheme in a multirate overlapped optical CDMA system," IEEE J. of Lightwave Technol., vol. 25, no. 8, pp. 2044 - 2053, August 2007.

[4] _ "Optical S-ALOHA/CDMA system for multirate applications: architecture, performance evaluation, and system stability," IEEE $J$. Lightwave Technol., vol. 24, no. 5, pp. 1968-1977, May 2006.

[5] E. Inaty, H. M. H. Shalaby, P. Fortier, and L. A. Rusch "Multirate optical fast frequency hopping CDMA system using power control," $J$. Lightwave Technol., vol. 20, pp. 166-177, February 2002.

[6] H. Yashima, and T. Kobayashi "Optical CDMA with time hopping and power control for multirate networks," J. Lightwave Technol., vol. 21, pp. 695-702, March 2003.

[7] T. Miyazawa and I. Sasase "Multi-rate and multi-quality transmission scheme using adaptive overlapping pulse-position modulator and power controller in optical network," IEEE ICON, vol. 1, pp. 127-131, November 2004.

[8] N. G. Tarhuni, M. S. Elmusrati, T. O. Korhonen, and E. Mutafungwa "Multi-access-interference mitigation using power control in opticalCDMA star networks," IEEE ICC, vol. 3, pp. 1593 - 1597, May 2005.

[9] J. Sherman and W. J. Morrison, "Adjustment of an Inverse Matrix Corresponding to a Change in One Element of a Given Matrix," Annals of Mathematical Statistics, Vol. 21, No. 1, pp. 124-127, 1950.

[10] L. Cooper. Applied Nonlinear Programming for Engineers and Scientists, 1974, Aloray, Englewood, New Jersey 07631 